\title{
Association of IRS2 overexpression with disease progression in intrahepatic cholangiocarcinoma
}

\author{
HUEY-LING YOU ${ }^{1,2^{*}}$, TING-TING LIU ${ }^{3 *}$, SHAO-WEN WENG ${ }^{4}$, CHANG-HAN CHEN $^{5}$, \\ YU-CHING WEI ${ }^{6}$, HOCK-LIEW ENG ${ }^{3}$ and WAN-TING HUANG ${ }^{1-3}$ \\ ${ }^{1}$ Department of Laboratory Medicine, Kaohsiung Chang Gung Memorial Hospital and \\ Chang Gung University College of Medicine, Kaohsiung 83301; ${ }^{2}$ Department of Medical Laboratory Sciences \\ and Biotechnology, Fooyin University, Kaohsiung 83102; Departments of ${ }^{3}$ Pathology and ${ }^{4}$ Internal Medicine; \\ ${ }^{5}$ The Institute for Translational Research in Biomedicine, Kaohsiung Chang Gung Memorial Hospital, \\ Kaohsiung 83301; ${ }^{6}$ Department of Pathology, Kaohsiung Municipal Ta-Tung Hospital, \\ Kaohsiung Medical University Hospital, Kaohsiung 80708, Taiwan, R.O.C.
}

Received January 11, 2018; Accepted July 27, 2018

DOI: $10.3892 / 01.2018 .9284$

\begin{abstract}
Insulin receptor substrate 2 (IRS2) is a candidate driver oncogene frequently amplified in cancer and is positively associated with IRS2 expression. The overexpression of IRS2 has been suggested to promote tumor metastasis. However, its function in intrahepatic cholangiocarcinoma (iCCA) has not been investigated extensively. The present study examined 86 cases of iCCA to analyze IRS2 expression and its correlation with clinicopathological characteristics using immunohistochemical assays. Three stable cell lines overexpressing IRS2 were established. The mobility potential of cells was compared in the basal condition and following manipulation using cell migration and invasion assays. Epithelial-mesenchymal transition (EMT)-associated proteins were assessed by western blotting. IRS2 was overexpressed in 29 iCCA cases $(33.7 \%)$ and was significantly more frequent in cases with large tumor size $(\mathrm{P}=0.033)$, classified as an advanced stage by the American Joint Committee on Cancer
\end{abstract}

Correspondence to: Dr Wan-Ting Huang, Department of Laboratory Medicine, Kaohsiung Chang Gung Memorial Hospital and Chang Gung University College of Medicine, 123 Ta-pei Road, Niao-Sung, Kaohsiung 83301, Taiwan R.O.C.

E-mail: huangwanting5@gmail.com

*Contributed equally

Abbreviations: IRS2, insulin receptor substrate 2; iCCA, intrahepatic cholangiocarcinoma; EMT, epithelial-mesenchymal transition; IGFs, insulin-like growth factors; IGFBs, IGF-binding proteins; DFS, disease-free survival; HCC, hepatocellular carcinoma; IHC, immunohistochemical; PyV-MT, polyomavirus middle $\mathrm{T}$ antigen

Key words: intrahepatic cholangiocarcinoma, IRS2, epithelial-mesenchymal transition, disease progression, disease-free survival
$(\mathrm{P}=0.046)$. In comparison with the control cells, the three IRS2-overexpressing iCCA cell lines exhibited a statistically significant increase in mobility potential. Expression analysis of EMT markers demonstrated decreased epithelial marker levels and increased mesenchymal marker levels in IRS2-overexpressing cells compared with their corresponding control cells. The results of the present study indicate that IRS2 overexpression is characterized by a large tumor size and advanced tumor stage in iCCA, and that it may increase tumor mobility potential by regulating EMT pathways. Therefore, it is a valuable predictive indicator of metastasis and may provide a novel direction for targeted therapy in iCCA.

\section{Introduction}

Insulin, insulin-like growth factors (IGFs), and IGF-binding proteins (IGFBs) are involved in numerous biological processes such as cellular growth, proliferation, metabolism, glucose homeostasis, cell differentiation, and apoptosis; they are implicated in the autocrine/paracrine stimulation of a variety of malignancies $(1,2)$. Insulin receptor substrates (IRSs) are adaptors of the insulin/IGF signaling pathways, with the ability to modulate and coordinate multiple signaling cascades, transmitting upstream signals to intracellular pathways, including the PI3K/AKT/mTOR and MAP kinase pathways (3). Deregulation of these pathways might increase the risk of several types of cancers, including prostate, colon, liver, pancreas, kidney, lung, and breast cancers (2,4-6). In vivo and in vitro experimental models have highlighted the role of increased insulin and IGF signaling in enhancing tumorigenesis (7). High IGF-1 and low IGFBP3 plasma levels indicate an increased risk of cancers (8). IGFBP3, a mediator of apoptosis, which has opposing effects to those of IGF1, can inhibit the growth of human breast cancer cells with HER 2 overexpression $(9,10)$.

IGF-1 receptors are widely distributed in many human malignancies (11). The upregulation of IGF-1 receptor signaling may contribute to resistance to therapies (12). 
Following the activation of IGF-1 receptors, IRSs are rapidly phosphorylated on tyrosine residues, revealing the docking site for multiple $\mathrm{SH}-2$-containing proteins such as p85, Nck, Crk, Fyn, Syp, and SHP2 at the carboxy terminus (13). The phosphorylation of IRSs leads to the transmission of mitogenic, anti-apoptotic, and anti-differentiation signals to tumor cells. Among the six family members of IRSs, IRS1 and IRS2 are widely expressed in human tissues (14). An increasing amount of evidence has revealed that they are involved in tumor progression, including cell proliferation, adhesion, and migration $(15,16)$. The pathological mechanisms contributing to malignancy could be a function of gene amplification, influenced by the feedback loops of other mutations and constitutively phosphorylated proteins. The overexpression of IRS1 or IRS2 may result in palpable tumors of the mammary glands, which exhibit a unique histopathology associated with the activation of $\beta$-catenin in murine models $(17,18)$. In breast cancer cells, the overexpression of IRS1 and IRS2 increases tumor proliferation and motility, respectively (19). IRS1 is implicated in IGF-mediated proliferation (15), whereas IRS2 is predominantly involved in tumor adhesion and migration following IGF-1 stimulation (3,15,19-21). The metastatic potential of tumors might be impeded by IRS deletion (22).

IRS2 has been reported to be overexpressed in hepatocellular carcinoma (HCC) as an early event or implicated in the later stages of tumorigenesis (4). IRS2 overexpression may promote the survival of tumor cells independently of IRS1 (4). The protective effect of IRS2 overexpression against apoptosis is also implicated in liver tumor progression. Our previous data have demonstrated that IRS2 overexpression is correlated with copy number amplification and associated with poor disease-free survival (DFS) in intrahepatic cholangiocarcinoma (iCCA) (23). By inducing IRS2 overexpression, the mobility potential of iCCA cells can be increased. However, further studies are needed to elucidate the role of IRS2 overexpression in migration and metastasis.

In the present study, we examined the clinicopathological features of IRS2 expression in 86 cases of iCCA and assessed the relationship between IRS2 overexpression and epithelial-mesenchymal transition (EMT) in cell lines. We aimed to examine the clinicopathological significance of IRS2 overexpression and whether this aberration correlates with EMT in iCCA.

\section{Materials and methods}

Tumor materials. We selected 86 formalin-fixed, paraffin-embedded tumor samples from the collection at the Department of Pathology, Chang Gung Memorial Hospital, (Kaohsiung, Taiwan). Then, we reviewed hematoxylin and eosin-stained slides and medical records, defined survival time, and constructed tissue microarrays for IRS2 immunostaining as previously described (23). The study was approved by the Institutional Review Board of Chang Gung Medical Foundation, in accordance with the Helsinki Declaration (IRB 103-0818C, 103-4961B).

Immunohistochemical (IHC) analysis. IHC staining was performed as previously described (23). The primary antibody against IRS2 (1:150; Abcam, Cambridge, UK) was used with the PicTureTM-Plus kit (ZYMED ${ }^{\circledR}$ 2nd Generation Polymer Detection System; Thermo Fisher Scientific, Inc., Waltham, MA, USA). The labeling intensities were 0 (negative), 1 (weak), 2 (moderate), and 3 (strong), and the percentages of tumor cells with cytoplasmic immunoreactivity for IRS2 were counted in $5 \%$ increments. The two scores were multiplied to calculate the expression index. Only cases containing two or more analyzable cores were scored, and the scores of multiple cores from the same patient were averaged to obtain the mean expression index. Whole sections were used for IHC staining in cases with non-informative tissue cores (no tumor, or analyzable cores <2). The expression index values were evaluated by two pathologists blinded to the clinicopathological data and averaged. After testing a series of cutoff values, the IRS2 protein was regarded as overexpressed when the expression index was $>150$.

Cell lines and stable transfection. The iCCA cell lines (RBE, SNU1079, and SSP25) were purchased from the Korean Cell Line Bank (Seoul, South Korea) and the Riken BRC Cell Bank (Koyadai, Japan). Tumor cell lines were cultured in RPMI medium (Gibco; Thermo Fisher Scientific, Inc.) as previously described (23). Cells were transfected with pCMV-IRS2 entry vector using Lipofectamine 2000 reagent (Invitrogen; Thermo Fisher Scientific, Inc.) according to the manufacturer's instructions. Then, the cells were selected with complete medium containing G418 (Sigma-Aldrich; Merck KGaA, Darmstadt, Germany). The medium was changed every fourth day. Positive clones were selected through resistance against G418 and characterized for DDK and IRS2 expression by western blot analysis.

Western blot analysis. Western blot analysis was performed with a sodium dodecyl sulfate-polyacrylamide gel electrophoresis system as previously described (23). Immunoblot analysis was performed by incubation with primary antibodies at $25^{\circ} \mathrm{C}$ for $2 \mathrm{~h}$ (Table I). The blots were then washed and incubated with a 1:2,000 dilution of horseradish peroxidase (HRP)-conjugated secondary antibody (Jackson, West Grove, PA, USA), followed by three washes with Tris-buffered saline-Tween. An enhanced chemiluminescent HRP substrate (Pierce, Rockford, IL, USA) was used for detection according to the manufacturer's instructions.

Cell migration and invasion assays. Cell migration and invasion were assessed as previously described (23). Briefly, $200 \mathrm{ml}$ of cell suspension was added to the top wells of the chamber with $8 \mu \mathrm{m}$ pores coated with $0.1 \mathrm{ml}$ of diluted Matrigel matrix coating solution (Corning Inc., Corning, NY, USA) for the invasion assay, or left uncoated for the migration assay. Average cell mobility was determined by counting three random high-powered fields at magnification, $\mathrm{x} 100$. Three independent experiments were performed for both invasion and migration assays.

Statistical analysis. All statistical analyses were performed using SPSS for Windows 17.0 software (SPSS Inc., Chicago, IL, USA). The significance of the association between IRS2 expression and histopathological variables was determined by chi-square and Fisher's exact tests. Comparisons between 
Table I. Description of western blot antibodies.

\begin{tabular}{lllc}
\hline Antibody & Vendor & Clone & WB (dilution) \\
\hline IRS2 & Abcam & Monoclonal & $1: 3,000$ \\
DDK & Origene & Monoclonal & $1: 2,000$ \\
E-cadherin & Upstate & Polyclonal & $1: 3,000$ \\
N-cadherin & Abcam & Polyclonal & $1: 3,000$ \\
Vimentin & Abcam & Monoclonal & $1: 3,000$ \\
Fibronectin & Abcam & Polyclonal & $1: 3,000$ \\
GAPDH & GeneTex & Polyclonal & $1: 10,000$ \\
\hline
\end{tabular}

the two groups were conducted using Student's t-test. Overall survival was calculated from the date of diagnosis to death as a result of all causes. DFS was computed from the time of surgery to recurrence in the liver or distant metastasis. The Kaplan-Meier method was used for univariate survival analysis, and the difference between survival curves was tested by a log-rank test. For all analyses, two-sided tests of significance were used with $\mathrm{P}<0.05$ considered to indicate a statistically significant difference.

\section{Results}

Correlation between IHC findings and clinicopathological variables. The cohort consisted of 48 men and 38 women, with a median age of 56.5 years (range, 30-84; mean, 56.8 years). Immunoexpression of the IRS2 protein was observed in 86 cases (Table II). IRS2 was overexpressed in 29 cases (33.7\%; Fig. 1) and was significantly more frequent in cases with large tumor size $(\mathrm{P}=0.033)$, classified as advanced stage by the American Joint Committee on Cancer $(\mathrm{P}=0.046)$. In addition, IRS2-overexpressing iCCA demonstrated a marginal difference in DFS. When IRS2 was overexpressed in the tumors, DFS was shorter (mean 31.8 vs. 51.1 months; $\mathrm{P}=0.302$ ).

IRS2 overexpression affected the migratory and invasive capacities of iCCA cells through EMT in vitro. To assess the oncogenic activity of IRS2 in cell lines, we established stable IRS2 expression in RBE, SNU1079, and SSP25 cells (designated as RBE-IRS2, SNU1079-IRS2, and SSP25-IRS2, respectively). IRS2-transfected cells were compared with the corresponding control cells with empty vectors; the number of migratory cells was significantly increased (RBE: $\mathrm{P}=0.007$; SNU1079: $\mathrm{P}=0.004$; SSP25: $\mathrm{P}=0.018$; Fig. 2). The penetration function of IRS2 during metastasis was assessed. The cell invasion ability increased following ectopic expression of IRS2 (RBE: P=0.006; SNU1079: $\mathrm{P}=0.003$; SSP25: $\mathrm{P}=0.019$; Fig. 2). In comparison with control cells, all the SSP25-IRS2 and SNU1079-IRS2 cells had higher numbers of migratory and invasive cells.

Furthermore, IRS2-trasfected cells lost intercellular cohesion and displayed a more spindle-like fibroblastic appearance (Fig. 3A). Expression analysis of EMT markers demonstrated decreased epithelial marker levels (E-cadherin) and increased mesenchymal marker levels ( $\mathrm{N}$-cadherin and fibronectin) in RBE-IRS2, SSP25-IRS2, and SNU1079-IRS2 cells compared with their corresponding control cells (Fig. 3B). The data
Table II. Clinicopathological characteristics and associations with IRS2 immunoexpression in 86 intrahepatic cholangiocarcinoma.

\begin{tabular}{|c|c|c|c|c|}
\hline \multirow[b]{2}{*}{ Parameters } & \multirow{2}{*}{$\begin{array}{l}\text { No. of } \\
\text { patients }\end{array}$} & \multicolumn{2}{|c|}{ IRS2 expression } & \multirow[b]{2}{*}{ P-value } \\
\hline & & Positive & Negative & \\
\hline Age, years & & & & NS \\
\hline$\leq 60$ & 49 & 19 & 30 & \\
\hline$>60$ & 37 & 10 & 27 & \\
\hline Sex & & & & NS \\
\hline Male & 48 & 13 & 35 & \\
\hline Female & 38 & 16 & 22 & \\
\hline Gross pattern & & & & NS \\
\hline $\mathrm{MF}$ & 52 & 14 & 38 & \\
\hline $\mathrm{MF}+\mathrm{PI}$ & 33 & 14 & 19 & \\
\hline Tumor N & & & & NS \\
\hline Solitary & 69 & 23 & 46 & \\
\hline Multiple & 16 & 6 & 10 & \\
\hline Tumor size, $\mathrm{cm}$ & & & & $0.033^{\mathrm{a}}$ \\
\hline 5 & 46 & 11 & 35 & \\
\hline$>5$ & 40 & 18 & 22 & \\
\hline Necrosis & & & & NS \\
\hline$\leq 10 \%$ & 62 & 24 & 38 & \\
\hline$>10 \%$ & 24 & 5 & 19 & \\
\hline VI & & & & NS \\
\hline No & 52 & 18 & 34 & \\
\hline Yes & 34 & 11 & 23 & \\
\hline NI & & & & NS \\
\hline No & 55 & 18 & 37 & \\
\hline Yes & 31 & 11 & 20 & \\
\hline $\mathrm{H}$ grade & & & & NS \\
\hline I & 26 & 9 & 17 & \\
\hline II+III & 60 & 20 & 40 & \\
\hline Stage & & & & $0.046^{\mathrm{a}}$ \\
\hline I+II & 45 & 11 & 34 & \\
\hline III+IV & 41 & 18 & 23 & \\
\hline
\end{tabular}

aStatistically significant. NS, not significant; M, mass-forming type; PI, periductal infiltrating type; $\mathrm{N}$, number; VI, vascular invasion; NI, neural invasion; H, histology; IRS2, insulin receptor substrate 2.

suggest that IRS2 overexpression could promote EMT despite the conflicting results of vimentin expression in RBE-IRS2 and SNU1079-IRS2 cells.

\section{Discussion}

In this study, we investigated the clinicopathological roles of IRS2 expression in 86 iCCA samples. IRS2 overexpression was associated with large tumor size and advanced tumor stage. Furthermore, the transfection of IRS2 in iCCA cells promoted migration, invasion, and EMT in vitro. Taking together all the findings, it can be surmised that IRS2 plays 

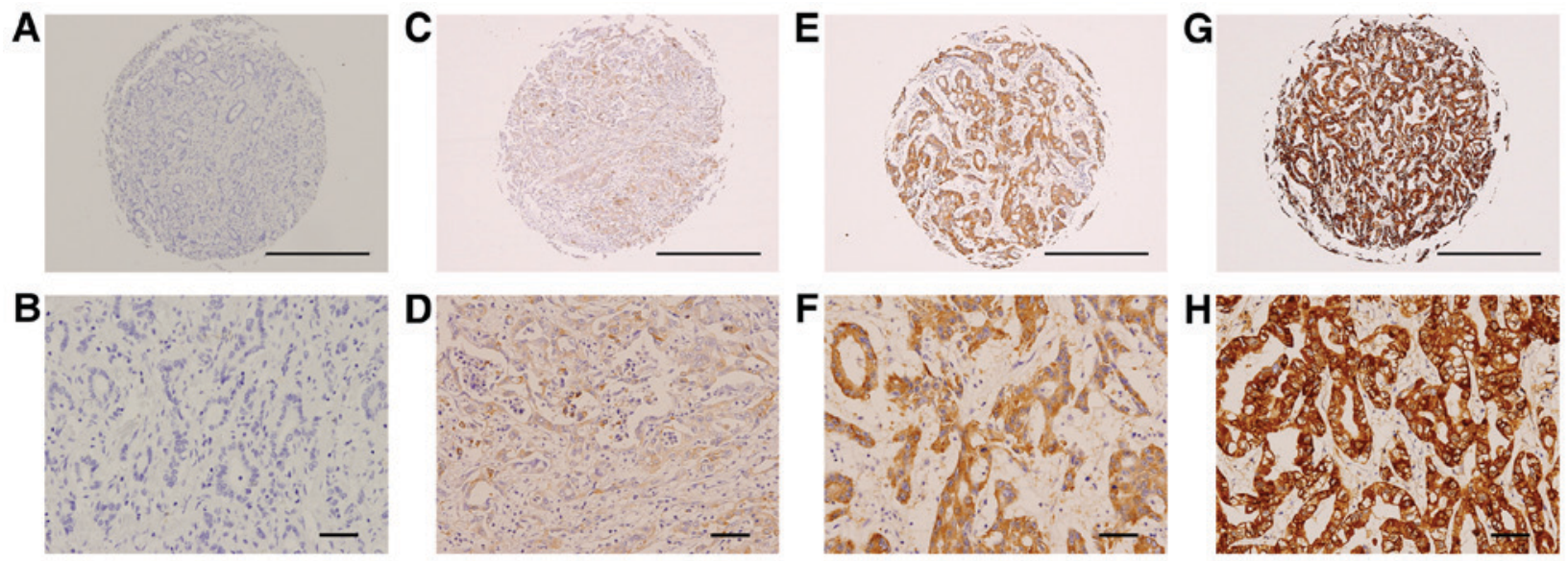

Figure 1. Representative photographs of IRS2 immunostaining in iCCA. (A, C, E, and G) Tissue microarray cores at magnification, $\mathrm{x} 40$ (scale bar, $500 \mu \mathrm{m}$ ); $(\mathrm{B}, \mathrm{D}, \mathrm{F}$, and $\mathrm{H})$ selected areas from $(\mathrm{C}, \mathrm{E}$, and $\mathrm{G})$ at a higher magnification (x200; scale bar, $50 \mu \mathrm{m})$. Expression index values were calculated by multiplying the percentage of positive tumor cells by the average intensity. (A and B) The negative control incubated with secondary antibody only. (C and D) Weak staining (1+) with 15\% positive tumor cells. (E and F) Moderate staining (2+) with 85\% positive tumor cells. (G and H) Strong staining (3+) with 95\% positive tumor cells. iCCA, intrahepatic cholangiocarcinoma.
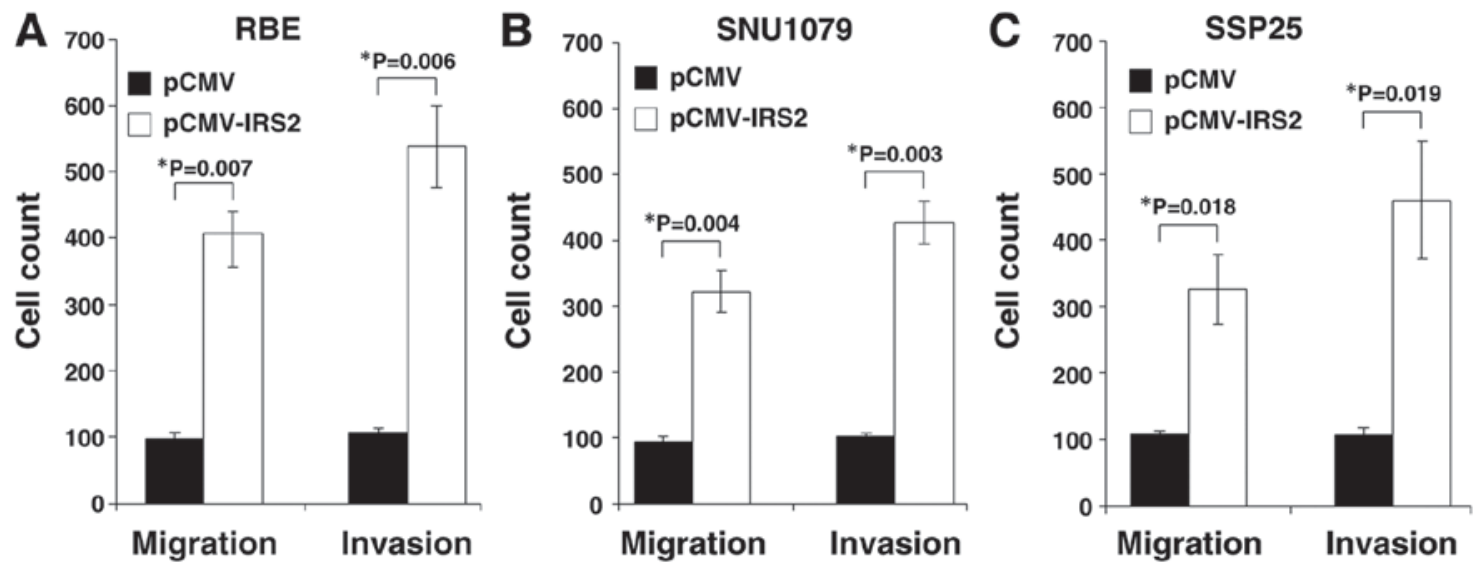

Figure 2. Enhancement of the mobility potential of iCCA cells by IRS2. The cell migration and cell invasion assays of pCMV-IRS2-transfected and empty vector-transfected (A) RBE, (B) SNU1079, and (C) SSP25 cell lines were compared. The number of cells migrating through the membrane and cells invading through the Matrigel of each cell line are presented. The results indicate that IRS2 can promote cell migration and cell invasion. Data represent the mean \pm standard deviation of three independent experiments. iCCA, intrahepatic cholangiocarcinoma; IRS2, insulin receptor substrate 2 .
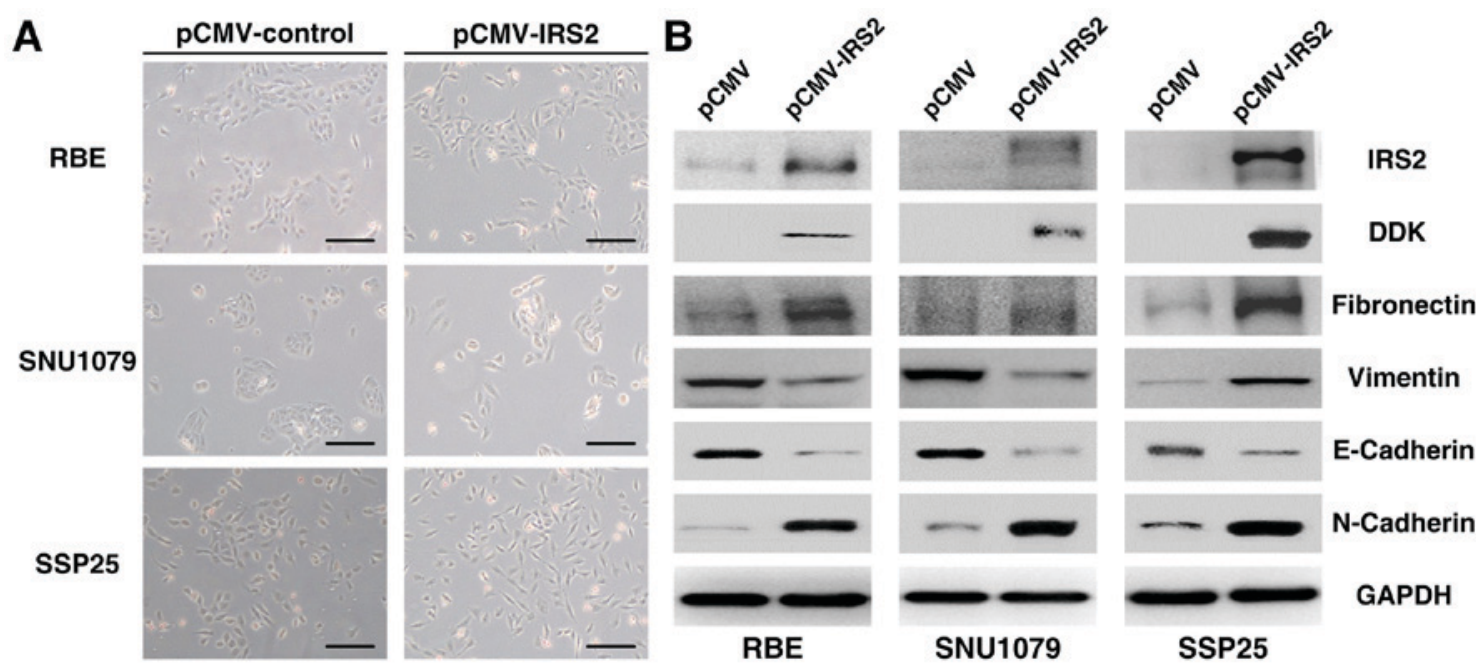

Figure 3. Effect of pCMV-IRS2 transfection on the EMT in RBE, SNU1079, and SSP25 cells. (A) After transfection with pCMV-IRS2 vectors, morphological changes from a cobblestone-like (left) to a spindle-like fibroblastic (right) appearance (magnification, x50; scale bar, $100 \mu \mathrm{m}$ ). (B) RBE, SNU1079, and SSP25 cell extracts were subjected to 10\% SDS-PAGE and western blot analysis with the respective primary antibodies against IRS2, vimentin, N-cadherin, fibronectin, and E-cadherin. GAPDH was used as an internal control. EMT, epithelial-mesenchymal transition; IRS2, insulin receptor substrate 2. 
a major role in regulating EMT in iCCA. The upregulation of IRS2 may be a late event, promoting tumor progression and possibly accounting for its more frequent presence in large-sized tumors.

The putative role of IRS2 in tumor metastasis is supported by other studies. Day et al demonstrated that an increase in IRS2 expression is associated with disease progression through the stages of colorectal carcinoma formation (15). A metastatic phenotype, conferring tumor cells with the ability to invade and survive in foreign environments, has been identified in IRS2-overexpressing mammary tumors (24). Therefore, the upregulation of IRS2 levels and activity may contribute to tumor metastasis (24), as opposed to the effects of IRS 2 gene deletion (22). The relationship between IRS2 expression and liver cancer has not been explored in detail. Boissan et al reported that high IRS2 levels can promote tumor survival and progression in HCC (4). Consistent with other studies, IRS2-overexpressing iCCA demonstrated increased migratory and invasive capacities, which may lead to an advanced tumor stage and adverse prognosis. Meanwhile, the knockdown of IRS 2 has been found to inhibit the mobility potential (23).

The present study revealed a function of IRS2 in iCCA metastasis through EMT regulation. For migration, cancer cells must first overcome programmed cell death and decrease proliferation rates when the contact with the surrounding extracellular matrix is lost. With the loss of the expression of E-cadherin, a key marker of the epithelial phenotype, followed by the expression of mesenchymal markers such as $\mathrm{N}$-cadherin, vimentin, and fibronectin, the invasive ability of cancer cells is increased. Several transcription factors are upregulated and involved in EMT; Snail, ZEB, and Twist induce epigenetic silencing at the E-cadherin promoter, decreasing E-cadherin expression in the development of the mesenchymal phenotype. Recently, large-scale evidence has revealed that IGF-I/IGF-IR signaling is involved in EMT-associated tumor metastasis and drug resistance (25-28). Our study showed that iCCA cells with stable IRS2 expression exhibited mesenchymal phenotypes characterized by the increased expression of $\mathrm{N}$-cadherin and fibronectin in all cell lines. Moreover, the expression of E-cadherin, responsible for the epithelial phenotype, was decreased. Thus, IRS2 overexpression is a factor involved in the regulation of EMT in iCCA mainly through $\mathrm{N}$-cadherin, fibronectin, and E-cadherin.

Ligand activation of IGF-IR activates two main signaling pathways, the IRS/PI3K/Akt and Ras/Raf/ERK pathways, which regulate the transcription factors of EMT, including members of ZEB, Snail, and Twist families. Crosstalk between other signaling pathways and IGF signaling are also involved in the EMT process, including the crosstalk and positive feedback loop between IGF-I signaling and Wnt/ $\beta$-catenin signaling and the activation of Notch signaling (29). As for the discrepancy of IRS2-induced change in vimentin expression between different cell lines, we speculate that the overexpression of IRS2 in different cell lines might have direct and indirect impacts on vimentin expression. IRS2 overexpression directly increased vimentin expression in the SSP25 cell line through the IRS/PI3K/Akt pathway, while IRS2 indirectly decreased vimentin through the crosstalk of the EMT pathway. Further investigation to disclose the genetic background related to the IRS/PI3K/Akt pathway and EMT pathway in different cell lines will help elucidate the discrepancy.

Although IRS2 is associated with metastatic behaviors, it is not required for tumor initiation and growth. By using the polyomavirus middle $\mathrm{T}$ antigen (PyV-MT) mouse model of mammary tumorigenesis, Nagle et al found no differences in mammary tumor onset or growth between Irs2-null and wild-type mice (22). The ability of PyV-MT-derived tumor cells to metastasize to the lungs was also significantly impaired (22). Despite the close structural homology of IRS1 with IRS2, IRS1 may regulate cancer metastasis in a different manner. Ma et al demonstrated that IRS1 expression was selectively inhibited in metastatic mammary tumors (24). IRS1 may function as a metastasis suppressor, given that IRS2 overexpression, as a compensation for the loss of IRS1, promotes mammary tumor metastasis (24). Similar malignant tumor phenotypes affected by IRS1 and IRS2 have been found in other tumor cells: HCC, neuroblastoma, mesothelioma, and prostate carcinoma $(4,30,31)$. Our data are in line with previous findings and indicate that IRS2 is a candidate prognostic marker for iCCA metastasis.

Considering that the insulin/IGF signaling pathways are commonly known for fine-tuning numerous biological processes in cancer, targeting these pathways is a promising strategy in cancer therapy. Some therapeutic strategies include small-molecule tyrosine kinase inhibitors, receptor blockage with neutralizing antibodies, and IGFBs, which have been developed and evaluated in preclinical and clinical phases (1). IGF-1R and IR tyrosine kinase inhibitors can inhibit constitutive or ligand-dependent phosphorylation, as well as the downstream PI3K/AKT/mTOR and Erk MAP kinase pathways $(32,33)$. Neutralizing antibodies are monoclonal antibodies developed to target the extracellular domain of IGF-1R and IR, subsequently blocking the downstream signaling pathways (1). Both strategies result in the inhibition of cell proliferation and stimulation of apoptosis in cancer cells (1). Recently described antibodies against IGF to neutralize the ligand have been found to be effective in inhibiting the tumor metastasis of a variety of cancer cells $(34,35)$. A unique family of small molecules, IRS-targeted agents that can lead to Ser phosphorylation and the destruction of IRS1 and IRS2, results in the long-term inhibition of IR/IGF-IR signaling and strong inhibition of tumor cell growth (12). Therefore, targeting IGF-1R and IR can be a promising strategy in cancer therapy.

In conclusion, our present results showed that iCCA patients with IRS2 overexpression had large tumor size and advanced tumor stage compared to patients without IRS2 overexpression. According to in vitro studies, the stable IRS2-introdued cells lines had a higher mobility potential, owing to the association with EMT pathways. The regulatory mechanisms between IRS2 expression and EMT pathways should be further investigated. We propose that IRS2 is a valuable predictive indicator of metastasis and may provide new directions for targeted therapy in iCCA.

\section{Acknowledgements}

Not applicable. 


\section{Funding}

The study was supported by the Ministry of Science and Technology, Taiwan (grant nos. MOST 104-2320-B-182A-010 and MOST 105-2320-B-182A-016) and Chang Gung Memorial Hospital (grant nos. CMRPG8C0591-2, CMRPG8E1471-2, CMRPG8B1251-3, CMRPG8D1321 and CMRPG8E0921). The funders had no role in the manuscript design, data collection and analysis, decision to publish, or preparation of the manuscript.

\section{Availability of data and materials}

All data generated or analyzed during this study are included in this published article.

\section{Author's contributions}

WTH conceived and designed the experiments. HLY, TTL and CHC performed the experiments. SWW, YCW and HLE analyzed the data. HLY and TTL wrote the manuscript. All authors have read and approved the final manuscript.

\section{Ethics approval and consent to participate}

The present study was approved by the Chang Gung Medical Foundation Institutional Review Board (approval no. IRB 103-0818C, 103-4961B). Informed consent for participation was waived by the International Review Board on the basis that all samples and medical data used in the present study has been irreversibly anonymized. Furthermore, it was a retrospective study using archived material.

\section{Patient consent for publication}

Not applicable.

\section{Competing interests}

The authors declare that they have no competing interests.

\section{References}

1. Singh P, Alex JM and Bast F: Insulin receptor (IR) and insulin-like growth factor receptor 1 (IGF-1R) signaling systems: Novel treatment strategies for cancer. Med Oncol 31: 805, 2014.

2. Kasper JS and Giovannucci E: A meta-analysis of diabetes mellitus and the risk of prostate cancer. Cancer Epidemiol Biomarkers Prev 15: 2056-2062, 2006.

3. Dearth RK, Cui X, Kim HJ, Hadsell DL and Lee AV: Oncogenic transformation by the signaling adaptor proteins insulin receptor substrate (IRS)-1 and IRS-2. Cell Cycle 6: 705-713, 2007.

4. Boissan M, Beurel E, Wendum D, Rey C, Lécluse Y, Housset C, Lacombe ML and Desbois-Mouthon C: Overexpression of insulin receptor substrate-2 in human and murine hepatocellular carcinoma. Am J Pathol 167: 869-877, 2005.

5. Esposito DL, Verginelli F, Toracchio S, Mammarella S, De Lellis L, Vanni C, Russo A, Mariani-Costantini R and Cama A: Novel insulin receptor substrate 1 and 2 variants in breast and colorectal cancer. Oncol Rep 30: 1553-1560, 2013.

6. Rozengurt E, Sinnett-Smith J and Kisfalvi K: Crosstalk between insulin/insulin-like growth factor-1 receptors and $G$ protein-coupled receptor signaling systems: A novel target for the antidiabetic drug metformin in pancreatic cancer. Clin Cancer Res 16: 2505-2511, 2010.
7. Hsing AW, Sakoda LC and ChuaS Jr: Obesity, metabolic syndrome, and prostate cancer. Am J Clin Nutr 86: s843-s857, 2007.

8. Dziadziuszko R, Camidge DR and Hirsch FR: The insulin-like growth factor pathway in lung cancer. J Thorac Oncol 3: 815-818, 2008.

9. Jerome L, Alami N, Belanger S, Page V, Yu Q, Paterson J, Shiry L, Pegram M and Leyland-Jones B: Recombinant human insulin-like growth factor binding protein 3 inhibits growth of human epidermal growth factor receptor-2-overexpressing breast tumors and potentiates herceptin activity in vivo. Cancer Res 66: 7245-7252, 2006.

10. Nahta R, Yu D, Hung MC, Hortobagyi GN and Esteva FJ: Mechanisms of disease: Understanding resistance to HER2-targeted therapy in human breast cancer. Nat Clin Pract Oncol 3: 269-280, 2006.

11. Ouban A, Muraca P, Yeatman T and Coppola D: Expression and distribution of insulin-like growth factor-1 receptor in human carcinomas. Hum Pathol 34: 803-808, 2003.

12. Reuveni H, Flashner-Abramson E, Steiner L, Makedonski K, Song R, Shir A, Herlyn M, Bar-Eli M and Levitzki A: Therapeutic destruction of insulin receptor substrates for cancer treatment. Cancer Res 73: 4383-4394, 2013.

13. Taniguchi CM, Emanuelli B and Kahn CR: Critical nodes in signalling pathways: Insights into insulin action. Nat Rev Mol Cell Biol 7: 85-96, 2006.

14. Cai D, Dhe-Paganon S, Melendez PA, Lee J and Shoelson SE: Two new substrates in insulin signaling, IRS5/DOK4 and IRS6/DOK5. J Biol Chem 278: 25323-25330, 2003.

15. Day E, Poulogiannis G, McCaughan F, Mulholland S, Arends MJ, Ibrahim AE and Dear PH: IRS2 is a candidate driver oncogene on 13q34 in colorectal cancer. Int J Exp Pathol 94: 203-211, 2013.

16. Yuan TL and Cantley LC: PI3K pathway alterations in cancer: Variations on a theme. Oncogene 27: 5497-5510, 2008.

17. Rosner A, Miyoshi K, Landesman-Bollag E, Xu X, Seldin DC, Moser AR, MacLeod CL, Shyamala G, Gillgrass AE and Cardiff RD: Pathway pathology: Histological differences between ErbB/Ras and Wnt pathway transgenic mammary tumors. Am J Pathol 161: 1087-1097, 2002.

18. Dearth RK, Cui X, Kim HJ, Kuiatse I, Lawrence NA, Zhang X, Divisova J, Britton OL, Mohsin S, Allred DC, et al: Mammary tumorigenesis and metastasis caused by overexpression of insulin receptor substrate 1 (IRS-1) or IRS-2. Mol Cell Biol 26: 9302-9314, 2006.

19. Byron SA, Horwitz KB, Richer JK, Lange CA, Zhang X and Yee D: Insulin receptor substrates mediate distinct biological responses to insulin-like growth factor receptor activation in breast cancer cells. Br J Cancer 95: 1220-1228, 2006.

20. Gibson SL, Ma Z and Shaw LM: Divergent roles for IRS-1 and IRS-2 in breast cancer metastasis. Cell Cycle 6: 631-637, 2007.

21. Mardilovich K and Shaw LM: Hypoxia regulates insulin receptor substrate-2 expression to promote breast carcinoma cell survival and invasion. Cancer Res 69: 8894-8901, 2009.

22. Nagle JA, Ma Z, Byrne MA, White MF and Shaw LM: Involvement of insulin receptor substrate 2 in mammary tumor metastasis. Mol Cell Biol 24: 9726-9735, 2004.

23. Liu TT, You HL, Weng SW, Wei YC, Eng HL and Huang WT: Recurrent amplification at $13 \mathrm{q} 34$ targets at CUL4A, IRS2, and TFDP1 As an independent adverse prognosticator in intrahepatic cholangiocarcinoma. PLoS One 10: e0145388, 2015.

24. Ma Z, Gibson SL, Byrne MA, Zhang J, White MF and Shaw LM: Suppression of insulin receptor substrate 1 (IRS-1) promotes mammary tumor metastasis. Mol Cell Biol 26: 9338-9351, 2006.

25. Zhao H, Desai V, Wang J, Epstein DM, Miglarese M and Buck E: Epithelial-mesenchymal transition predicts sensitivity to the dual IGF-1R/IR inhibitor OSI-906 in hepatocellular carcinoma cell lines. Mol Cancer Ther 11: 503-513, 2012.

26. Denduluri SK, Idowu O, Wang Z, Liao Z, Yan Z, Mohammed M, Ye J, Wei Q, Wang J, Zhao L and Luu HH: Insulin-like growth factor (IGF) signaling in tumorigenesis and the development of cancer drug resistance. Genes Dis 2: 13-25, 2015.

27. Motallebnezhad M, Aghebati-Maleki L, Jadidi-Niaragh F, Nickho H, Samadi-Kafil H, Shamsasenjan K and Yousefi M: The insulin-like growth factor-I receptor (IGF-IR) in breast cancer: Biology and treatment strategies. Tumour Biol 37: 11711-11721, 2016.

28. Wang R, Li H, Guo X, Wang Z, Liang S and Dang C: IGF-I induces epithelial-to-mesenchymal transition via the IGF-IR -Src-MicroRNA-30a-E-cadherin pathway in nasopharyngeal carcinoma cells. Oncol Res 24: 225-231, 2016. 
29. Li H, Batth IS, Qu X, Xu L, Song N, Wang R and Liu Y: IGF-IR signaling in epithelial to mesenchymal transition and targeting IGF-IR therapy: Overview and new insights. Mol Cancer 16: 6, 2017.

30. Hoang CD, Zhang X, Scott PD, Guillaume TJ, Maddaus MA, Yee D and Kratzke RA: Selective activation of insulin receptor substrate-1 and -2 in pleural mesothelioma cells: Association with distinct malignant phenotypes. Cancer Res 64: 7479-7485, 2004.

31. Kim B, van Golen CM and Feldman EL: Insulin-like growth factor-I signaling in human neuroblastoma cells. Oncogene 23: 130-141, 2004.

32. Wittman M, Carboni J, Attar R, Balasubramanian B, Balimane P, Brassil P, Beaulieu F, Chang C, Clarke W, Dell J, et al: Discovery of a (1H-benzoimidazol-2-yl)-1H-pyridin-2-one (BMS-536924) inhibitor of insulin-like growth factor I receptor kinase with in vivo antitumor activity. J Med Chem 48: 5639-5643, 2005
33. Pitts TM, Tan AC, Kulikowski GN, Tentler JJ, Brown AM, Flanigan SA, Leong $S$, Coldren CD, Hirsch FR, Varella-Garcia $\mathrm{M}$, et al: Development of an integrated genomic classifier for a novel agent in colorectal cancer: Approach to individualized therapy in early development. Clin Cancer Res 16: 3193-3204, 2010.

34. Miyamoto S, Nakamura M, Shitara K, Nakamura K, Ohki Y, Ishii G, Goya M, Kodama K, Sangai T, Maeda H, et al: Blockade of paracrine supply of insulin-like growth factors using neutralizing antibodies suppresses the liver metastasis of human colorectal cancers. Clin Cancer Res 11: 3494-3502, 2005.

35. Goya M, Miyamoto S, Nagai K, Ohki Y, Nakamura K, Shitara K, Maeda H, Sangai T, Kodama K, Endoh Y, et al: Growth inhibition of human prostate cancer cells in human adult bone implanted into nonobese diabetic/severe combined immunodeficient mice by a ligand-specific antibody to human insulin-like growth factors. Cancer Res 64: 6252-6258, 2004. 